\section{Microbiological and physicochemical properties of smoked ricotta cheese during refrigeration and temperature abuse storage}

\author{
Christian Scarano, ${ }^{1}$ Carlo Spanu, ${ }^{1}$ \\ Anna Maria Mocci, ${ }^{1}$ Francesca Piras, ${ }^{1}$ \\ Mariella Demontis, ${ }^{1}$ Gavino Murittu, ${ }^{2}$ \\ Giuliano Pinna, ${ }^{2}$ Angela Santoru, ${ }^{2}$ \\ Enrico Pietro Luigi De Santis ${ }^{1}$ \\ ${ }^{1}$ Department of Veterinary Medicine, \\ University of Sassari; ${ }^{2}$ F.lli Pinna Dairy \\ Industry, Thiesi (SS), Italy
}

\section{Abstract}

In the last years changes occurred in the production process of ricotta mustia, a traditional smoked, salted and sometimes ripened ricotta cheese, produced in Sardinia. Fresher, slightly smoked and with reduced salt content products, were introduced into the market to meet changes in consumer's preferences for milder products. The present study of durability was conducted on an innovative fresh and smoked industrial product, also characterized by the small size and the packaging in modified atmosphere. A durability test to assess the evolution of microbiological and physicochemical profile of the product stored at refrigeration $\left(4^{\circ} \mathrm{C}\right)$ and mild abuse $\left(7^{\circ} \mathrm{C}\right)$ temperatures was carried out. A total of 126 ricotta samples smoked for either 1,2 , or 3 $\mathrm{h}$ were analyzed at intervals during shelflife for the determination of aerobic mesophilic counts, Enterobacteriaceae, yeast, moulds, $L$. monocytogenes, Pseudomonas spp. and B. cereus. Intrinsic properties, physic-chemical and headspace gas composition were also analyzed. Average and standard deviation were respectively $6,06 \pm 0,22$ for $\mathrm{pH}, 0,982 \pm 0,05$ for $\mathrm{a}_{\mathrm{W}}, 74,67 \% \pm 1,81 \%$ for moisture, $10,25 \% \pm 1,35 \%$ for fat, $10,92 \% \pm 0,46 \%$ for protein and $1,70 \% \pm 0,42 \%$ for salt content. Total bacterial count ranged between $3.88 \pm 0.48 \mathrm{log} \mathrm{cfu} / \mathrm{g}$ at $\mathrm{T}_{0}$ and $3.25 \pm 1.02$ at $\mathrm{T}_{45}$. L. monocytogenes, Pseudomonas spp. and $E$. coli were always below the detection limit. Enterobacteriaceae prevalence (percentage) was $3.17 \%(2.62 \pm 0.42 \lg 10 \mathrm{cfu} / \mathrm{g})$ and was limited to samples stored longer than 30 days while $B$. cereus was recovered in $5.55 \%(2.36 \pm 0.35 \lg 10 \mathrm{cfu} / \mathrm{g})$ of the samples and was never observed in samples after 45 days of refrigerated storage. The durability study is preliminary to challenge test to assess the shelf-life of this product in compliance with the requirements of Regulation (EC) 2073/2005.

\section{Introduction}

Whey is the fluid product obtained during the manufacture of cheese, casein or similar products by separation from the curd after coagulation of milk and/or of products obtained from milk (CODEX STAN 289-1995). The main food use of whey is the preparation of whey cheeses, whey drinks and fermented whey drinks (FAO, 2018). Ricotta is a general term used to identify a variety of Italian whey cheese. The manufacturing of ricotta cheese is based on the heat-denaturation of whey proteins up to cause their coagulation. Especially in the Mediterranean basin, there are several traditional ovine whey cheeses manufactured after the production of sheep milk cheeses such as Mizithra, Anthotyros and Manouri (Greece), Anari (Cyprus), Requesón (Spain), Requeijao (Portugal), Brocciu (France), Urdă (Balkans region) (Casti et al., 2016). Sardinia is the Italian region leader for dairy sheep industry (Storelli et al., 2012). Typical production from sheep milk whey consist of fresh (ricotta fresca), salted (ricotta toscanella, ricotta testa di morto and ricotta moliterna) or salted, smoked and sometimes ripened (ricotta mustia) ricotta cheese. These products are included in the "List of Traditional Agri-Food Products" of the Italian Ministry of Agricultural, Food and Forestry Policies (Ministerial Decree 18 luglio 2000). Smoked ricotta cheese is manufactured in artisanal (Ricotta mustia) or industrial cheesemaking plants (ricotta toscanella) by pressing the curd to enhance drainage, then dry salted and smoked, at artisanal level by combustion of aromatic woods in a fireplace while at industrial level into a smoking chamber. Changes in consumer's lifestyle and preferences has driven food business operators to place new ricotta cheese product on the market, competing with local foods. In Sardinia, the ricotta production process has already been implemented, both at industrial and at artisanal level, including innovation steps such as MAP packaging, use of protective cultures to control secondary contaminations (Pala et al., 2016; Spanu et al., 2017, 2018) or the use of lactase to reduce the lactose content (Pulinas et al., 2017) in ricotta fresca, postlethality thermal treatment in ricotta salata (Spanu et al., 2013, 2015a,b). A possible strategy of product innovation is the modernization of traditional food processes and products (McElhatton and El Idrissi, 2016). Innovation of traditional foods can be
Correspondence: Carlo Spanu, Via Vienna 2, 07100, Sassari.

Tel.: +39.079.229425 - Fax: +39.079.229458. E-mail: cspanu@uniss.it

Key words: traditional products, sheep milk, process innovation, durability.

Contributions: the authors contributed equally.

Conflict of interest: the authors declare no potential conflict of interest.

Funding: none.

Received for publication: 19 December 2018. Revision received: 3 March 2019.

Accepted for publication: 12 April 2019.

This work is licensed under a Creative Commons Attribution-NonCommercial 4.0 International License (CC BY-NC 4.0).

(C) Copyright C. Scarano et al., 2019

Licensee PAGEPress, Italy

Italian Journal of Food Safety 2019; 8:8009

doi:10.4081/ijfs.2019.8009

obtained by changing the manufacturing process through the introduction of production steps, changes in product composition, packaging, product size and form or new ways to use the product (Gellynck and Kühne, 2008; Lipan et al., 2017). Following these trends, in recent years, has been observed a rise in the demand of small sized or single serving packaged ricotta cheese (Troiani, 2015). Some changes occurred also in the traditional smoked ricotta salata cheese (ricotta mustia) produced in Sardinia, with a fresher, slightly smoked and with reduced salt content products, introduced into the market to meet changes in consumer's preferences. With the purpose of meeting consumer's demand for fresh and healthy dairy products combined with the sensory attribute of the traditional product, an industrial sheep cheese making plant located in Sardinia (Italy) developed a product innovation of the traditional ricotta mustia. The innovated product, a fresh small sized, slightly salted ricotta cheese in MAP packaging, was developed to meet the GDO demand. In further sections of this paper, it will be referred as novel smoked ricotta cheese (NS-Ricotta Cheese). However, any time major process or formulation changes occurs, prior to place the food on the market, food business operator who aim to produce safe, wholesome and attractive food products, should conduct a proper shelf-life evaluation (Man and Jones, 1994).

The objective of the present study was to evaluate the impact of smoking time and 
temperature of storage on the microbiological profile and physicochemical properties of smoked ricotta cheese during shelf life. With this aim a durability study was conducted on MAP packed NS-Ricotta samples stored at refrigeration $\left(4^{\circ} \mathrm{C}\right)$ and mild abuse $\left(7^{\circ} \mathrm{C}\right)$ temperature for up to 45 days.

\section{Materials and Methods}

\section{NS-Ricotta Cheese samples}

The study was conducted in collaboration with an industrial sheep's milk cheese making plant located in Sardinia (Italy) which has developed and produced the NSRicotta Cheese samples used in the present experiment. Three NS-Ricotta Cheese batches were manufactured in different production days according to the following production steps. The whey remaining after the daily cheese production was filtered to remove curd residues and stored in a stainless silo tank until use. The whey was cleaned by centrifugal separators before being preheated to $60-70^{\circ} \mathrm{C}$ passing through a plate heat exchanger and then transferred into open kettles of 1,200-1500 1 of capacity. The whey was heated using a direct steam injection system until the temperature reached ca. $80^{\circ} \mathrm{C}$. The foam produced during heating was removed from the whey surface. As the flocculated proteins started to rise, heating was interrupted and the mixture held in the vat for ca. five minutes. Clots were then collected with the use of perforated ladles and transferred into plastic basket placed on drainage tables. Ricotta basket were allowed to drain and to cool for about ten-fifteen minutes after which the inner temperature of the curd dropped from $72-75^{\circ} \mathrm{C}$ to ca. $65-70^{\circ} \mathrm{C}$. Ricotta basket was transferred in a cold room at $4^{\circ} \mathrm{C}$ for $18-20$ hours. After refrigeration, the ricotta cheese was dry salted and cold smoked $\left(25-30^{\circ} \mathrm{C}\right)$ in a smoking chamber for either one, two or three hours. The smoke was produced by burning of beech (Fagus sylvatica) shavings. After $24 \mathrm{~h}$ of refrigerated storage $\left(4^{\circ} \mathrm{C}\right)$ in cold room, smoked ricotta cheese was packed in modified atmosphere using rigid polypropylene trays sealed with highbarrier peelable laminated films. The gas mixture used was $90 \% \mathrm{~N}$ and $10 \% \mathrm{CO}_{2}$. The final product was a truncated cone shaped cheese with upper base ca. $6.5 \mathrm{~cm}$ wide, lower base ca. $8.5 \mathrm{~cm}$ wide, height of ca. 6 $\mathrm{cm}$ and weigh of approximately $300 \mathrm{~g}$. The main differences in the manufacturing process of the NS-Ricotta Cheese as compared to ricotta mustia or toscanella are the absence of the pressing step, the cold smoking instead of hot smoking, the smaller size and weight (300 g vs. 800-2,000 g) and MAP packaging instead of food wrapping paper. The flowchart of the NS-Ricotta Cheese making process is represented in Figure 1. After packaging samples were stored refrigerated until analysis were performed.

\section{Experimental design}

A total of 126 NS-Ricotta Cheese samples were used, 42 from each of three production batches. From each batch fourteen ricotta samples were smoked for each of the following smoking time: 1,2 and 3 hours (H1, H2 and H3). The durability study was conducted analyzing NS-Ricotta Cheese samples at different moment during the shelf-life. Sampling times were: within $24 \mathrm{~h}$ after packaging, defined as time zero (T0), time 15 (T15), time 30 (T30) and time 45 (T45), respectively 15 days, 30 days and 45 days after packaging. In order to account for temperature abuse during the storage period, for each batch, for each smoking time and at each time point, duplicate ricotta sample were stored both at $4^{\circ} \mathrm{C}$ and at $7^{\circ} \mathrm{C}$. At T0 samples were kept only at $4^{\circ} \mathrm{C}$ with no thermal abuse. Table 1 reports the number of samples and the analysis performed at each sampling time.

\section{Microbiological analysis}

The preparation of the initial suspension and decimal dilution for microbiological examination was conducted according to ISO 6886-1:1999. From each sample two 25 $\mathrm{g}$ aliquots were aseptically collected and homogenized, one with $225 \mathrm{~mL}$ of Fraser Broth Base FBB (Biolife, Milan, Italy) for the detection of $L$. monocytogenes and one with $225 \mathrm{~mL}$ of Buffered Pepton Water BPW (Biolife) for all other parameters. After homogenization, serial decimal dilutions were prepared in BPW solution and used to

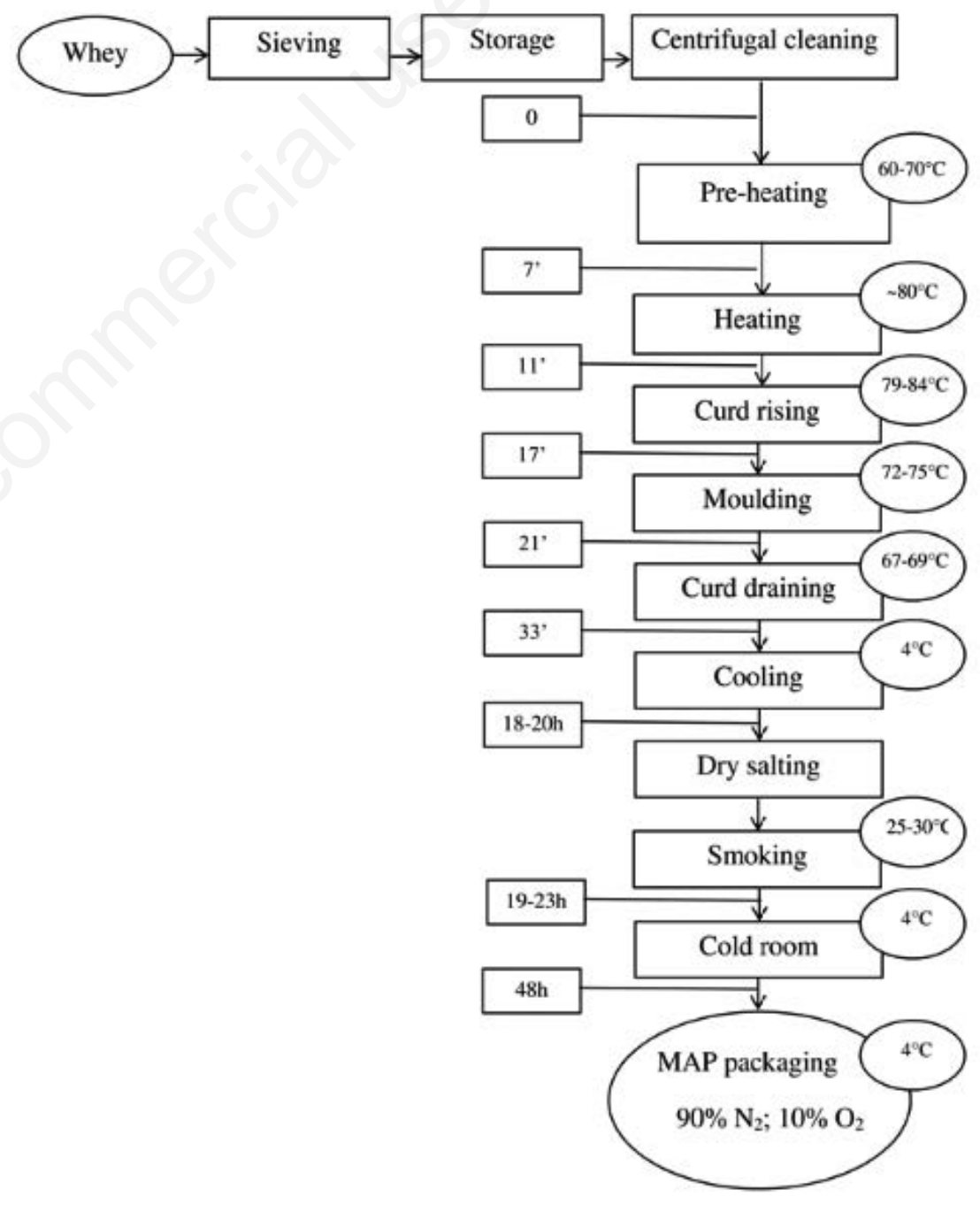

Figure 1. Flowchart of the NS-Ricotta Cheese making process. 
inoculate the appropriate culture media. The pour-plating procedure was used for the enumeration of aerobic mesophilic counts (ISO 4833; ISO, 2003), Enterobacteriaceae (ISO 21528-2; ISO, 2004), yeast and moulds (ISO 6611/IDF 94, 2004). The spread plating technique was used for the enumeration of $L$. monocytogenes (ISO 11290-1/2; ISO 1996, 1998), Pseudomonas spp (ISO/TS 11059:2009; ISO, 2009) and B. cereus (ISO 7932; ISO 2004).

\section{Physicochemical analysis and com- position}

Intrinsic properties such as $\mathrm{PH}$ and $\mathrm{a}_{\mathrm{w}}$ were measured using $\mathrm{pH}$ meter GLP22 (Crison Instruments, Barcelona, Spain) and water activity meter Aqualab 4TE
(Decagon, Pullman, WA, USA), respectively. Fat, moisture, protein and total solids were analyzed by using the compositional FoodScan $^{\mathrm{TM}}$ device (FOSS, Analytic, Hillerød, Denmark), which uses the nearinfrared spectrophotometer system.

\section{Headspace gas composition}

The determination of the headspaces gas composition was conducted on sealed NS-Ricotta Cheese samples before performing other analysis. Measures were obtained piercing the lid using a sterile needle connected to the Dansensor gas analyser (PBI Dansensor, Ringsted, Denmark). To avoid gas leaks during the penetration of needle, $15 \varnothing \mathrm{mm}$ septum (PBI Dansensor), were applied on the film lid before meas- urements of headspace gas composition. Measures of combined residual $\mathrm{O}_{2} \%$ and $\mathrm{CO}_{2} \%$ were directly read on the instrument while $\mathrm{N}_{2}$ was calculated by difference.

\section{Statistical analysis}

Differences among mean microbiological counts ( $\left.\mathrm{cfu} \mathrm{g}^{-1}\right)$, headspace gas concentration (\%), intrinsic properties $\left(\mathrm{pH}\right.$ and $\left.\mathrm{a}_{\mathrm{W}}\right)$ and centesimal composition (\%) over time $\left(\mathrm{T}_{0}, \mathrm{~T}_{15}, \mathrm{~T}_{30}\right.$ and $\left.\mathrm{T}_{45}\right)$ were compared using Fisher's least significant difference (LSD) test using Statgraphics Centurion XVI software (Stat Point Technologies, Warrenton, VA, USA). To account for the effect of smoking time (three levels: 1, 2 and 3 hours), storage temperature (two levels: $4^{\circ} \mathrm{C}$ and $7^{\circ} \mathrm{C}$ ) and storage duration (4 levels:

Table 1. Number of NS-Ricotta Cheese samples and analysis performed at each day of storage by length of smoking.

\begin{tabular}{|c|c|c|c|c|c|c|c|c|}
\hline \multirow[t]{3}{*}{ Parameters } & \multirow[t]{3}{*}{ Smoking ${ }^{4}$} & \multicolumn{5}{|c|}{ Day of storage } & \multirow{2}{*}{\multicolumn{2}{|c|}{$\mathrm{T}_{45}$}} \\
\hline & & $\mathrm{T}_{0}$ & & & & & & \\
\hline & & $4^{\circ} \mathrm{C}$ & $4^{\circ} \mathrm{C}$ & $7^{\circ} \mathrm{C}$ & $4^{\circ} \mathrm{C}$ & $7^{\circ} \mathrm{C}$ & $4^{\circ} \mathrm{C}$ & $7^{\circ} \mathrm{C}$ \\
\hline \multirow[t]{3}{*}{ Microbiological profile ${ }^{1}$, Physico-chemica' ${ }^{12}$ and headspace gas ${ }^{3}$ composition } & $\mathrm{H}_{1}$ & 6 & 6 & 6 & 6 & 6 & 6 & 6 \\
\hline & $\mathrm{H}_{2}$ & 6 & 6 & 6 & 6 & 6 & 6 & 6 \\
\hline & $\mathrm{H}_{3}$ & 6 & 6 & 6 & 6 & 6 & 6 & 6 \\
\hline
\end{tabular}

${ }^{1}$ Total aerobic mesophilic counts, Enterobacteriaceae, E. coli, L. monocytogenes, B. cereus, Pseudomonas spp, yeast and moulds; ${ }^{2} \mathrm{pH}$, aW, moisture $\%$, fat $\%$, protein $\%$ and salt $\% ;{ }^{3} \mathrm{O}_{2} \%$, $\mathrm{CO}{ }_{2} \%$ and $\mathrm{N}_{2} \% ;{ }^{4} \mathrm{Smoking}$ time hour (H): 1,2 and 3 .

Table 2. Evolution of the microbiological profile $\left(\log _{10} \mathrm{cfu} / \mathrm{g} ; \bar{x} \pm \mathrm{SD}\right)$ of MAP NS-Ricotta Cheese by smoking time during storage at $4^{\circ} \mathrm{C}$.

\begin{tabular}{|c|c|c|c|c|c|}
\hline \multirow[t]{2}{*}{ Microbial group } & \multirow[t]{2}{*}{ Smoking } & \multicolumn{4}{|c|}{ Day of storage } \\
\hline & & $\mathrm{T}_{0}$ & $\mathrm{~T}_{15}$ & $\mathrm{~T}_{30}$ & $\mathrm{~T}_{45}$ \\
\hline Aerobic mesophilic bacteria & $\begin{array}{l}\mathrm{H}_{1} \\
\mathrm{H}_{2} \\
\mathrm{H}_{3}\end{array}$ & $\begin{array}{l}(\mathrm{n}=6 / 6) 4.05 \pm 0.23^{\mathrm{al}} \\
(\mathrm{n}=6 / 6) 3.56 \pm 0.74^{\mathrm{al}} \\
(\mathrm{n}=6 / 6) 4.04 \pm 0.15^{\mathrm{al}}\end{array}$ & $\begin{array}{l}(\mathrm{n}=6 / 6) 2.82 \pm 0.36^{\mathrm{cl}} \\
(\mathrm{n}=6 / 6) 2.76 \pm 0.51^{\mathrm{abl}} \\
(\mathrm{n}=6 / 6) 2.32 \pm 0.32^{\mathrm{bl}}\end{array}$ & $\begin{array}{l}(\mathrm{n}=6 / 6) 3.15 \pm 0.83^{\mathrm{bcl}} \\
(\mathrm{n}=6 / 6) 2.69 \pm 0.96^{\mathrm{ab} 1} \\
(\mathrm{n}=6 / 6) 2.76 \pm 0.74^{\mathrm{bl}}\end{array}$ & $\begin{array}{l}(\mathrm{n}=6 / 6) 3.78 \pm 1.09^{\mathrm{abl}} \\
(\mathrm{n}=6 / 6) 2.68 \pm 0.61^{\mathrm{bl}} \\
(\mathrm{n}=6 / 6) 2.88 \pm 0.51^{\mathrm{bl}}\end{array}$ \\
\hline Enterobacteriaceae & $\begin{array}{l}\mathrm{H}_{1} \\
\mathrm{H}_{2} \\
\mathrm{H}_{3}\end{array}$ & $\begin{array}{l}(n=0 / 6) \text { N.D. } \\
(n=0 / 6) \text { N.D. } \\
(n=0 / 6) \text { N.D. }\end{array}$ & $\begin{array}{l}(\mathrm{n}=0 / 6) \text { N.D. } \\
(\mathrm{n}=0 / 6) \text { N.D. } \\
(\mathrm{n}=0 / 6) \text { N.D. }\end{array}$ & $\begin{array}{l}(n=0 / 6) \text { N.D. } \\
(n=0 / 6) \text { N.D. } \\
(n=0 / 6) \text { N.D. }\end{array}$ & $\begin{array}{c}(\mathrm{n}=1 / 6) 2.77 \pm 0.0 \\
(\mathrm{n}=0 / 6) \text { N.D. } \\
(\mathrm{n}=0 / 6) \text { N.D. }\end{array}$ \\
\hline E. coli & $\begin{array}{l}\mathrm{H}_{1} \\
\mathrm{H}_{2} \\
\mathrm{H}_{3}\end{array}$ & $\begin{array}{l}(n=0 / 6) \text { N.D. } \\
(n=0 / 6) \text { N.D. } \\
(n=0 / 6) \text { N.D. }\end{array}$ & $\begin{array}{l}(\mathrm{n}=0 / 6) \text { N.D. } \\
(\mathrm{n}=0 / 6) \text { N.D. } \\
(\mathrm{n}=0 / 6) \text { N.D. }\end{array}$ & $\begin{array}{l}(\mathrm{n}=0 / 6) \text { N.D. } \\
(\mathrm{n}=0 / 6) \text { N.D. } \\
(\mathrm{n}=0 / 6) \text { N.D. }\end{array}$ & $\begin{array}{l}(\mathrm{n}=0 / 6) \text { N.D. } \\
(\mathrm{n}=0 / 6) \text { N.D. } \\
(\mathrm{n}=0 / 6) \text { N.D. }\end{array}$ \\
\hline Yeast & $\begin{array}{l}\mathrm{H}_{1} \\
\mathrm{H}_{2} \\
\mathrm{H}_{3}\end{array}$ & $\begin{array}{l}(n=0 / 6) \text { N.D. } \\
(n=0 / 6) \text { N.D. } \\
(n=0 / 6) \text { N.D. }\end{array}$ & $\begin{array}{c}(\mathrm{n}=1 / 6) 1.30 \pm 0.0^{\mathrm{a}} \\
(\mathrm{n}=1 / 6) 1.60 \pm 0.0^{\mathrm{a}} \\
(\mathrm{n}=0 / 6) \text { N.D. }\end{array}$ & $\begin{array}{l}(\mathrm{n}=3 / 6) 2.78 \pm 0.68^{\mathrm{a}} \\
(\mathrm{n}=1 / 6) 2.00 \pm 0.0^{\mathrm{a}} \\
(\mathrm{n}=1 / 6) 3.08 \pm 0.0^{\mathrm{a}}\end{array}$ & $\begin{array}{c}(n=1 / 6) 3.08 \pm 0.0^{a} \\
(n=0 / 6) \text { N.D. } \\
(n=2 / 6) 3.04 \pm 0.19^{a}\end{array}$ \\
\hline Molds & $\begin{array}{l}\mathrm{H}_{1} \\
\mathrm{H}_{2} \\
\mathrm{H}_{3}\end{array}$ & $\begin{array}{l}(n=0 / 6) \text { N.D. } \\
(n=0 / 6) \text { N.D. } \\
(n=0 / 6) \text { N.D. }\end{array}$ & $\begin{array}{l}(n=0 / 6) \text { N.D. } \\
(n=0 / 6) \text { N.D. } \\
(n=0 / 6) \text { N.D. }\end{array}$ & $\begin{array}{l}(\mathrm{n}=3 / 6) 2.26 \pm 0.24^{\mathrm{a}} \\
(\mathrm{n}=1 / 6) 2.48 \pm 0.0^{\mathrm{a}} \\
(\mathrm{n}=2 / 6) 2.65 \pm 0.49^{\mathrm{a}}\end{array}$ & $\begin{array}{c}(\mathrm{n}=1 / 6) 3.00 \pm 0.0^{\mathrm{a}} \\
(\mathrm{n}=0 / 6) \text { N.D. } \\
(\mathrm{n}=1 / 6) 2.47 \pm 0.0^{\mathrm{a}}\end{array}$ \\
\hline Pseudomonas spp & $\begin{array}{l}\mathrm{H}_{1} \\
\mathrm{H}_{2} \\
\mathrm{H}_{3}\end{array}$ & $\begin{array}{l}(\mathrm{n}=0 / 6) \text { N.D. } \\
(\mathrm{n}=0 / 6) \text { N.D. } \\
(\mathrm{n}=0 / 6) \text { N.D. }\end{array}$ & $\begin{array}{l}(\mathrm{n}=0 / 6) \text { N.D. } \\
(\mathrm{n}=0 / 6) \text { N.D. } \\
(\mathrm{n}=0 / 6) \text { N.D. }\end{array}$ & $\begin{array}{l}(n=0 / 6) \text { N.D. } \\
(n=0 / 6) \text { N.D. } \\
(n=0 / 6) \text { N.D. }\end{array}$ & $\begin{array}{l}(n=0 / 6) \text { N.D. } \\
(n=0 / 6) \text { N.D. } \\
(n=0 / 6) \text { N.D. }\end{array}$ \\
\hline Bacillus cereus & $\begin{array}{l}\mathrm{H}_{1} \\
\mathrm{H}_{2} \\
\mathrm{H}_{3}\end{array}$ & $\begin{array}{c}(n=0 / 6) \text { N.D. } \\
(n=1 / 6) 2.60 \pm 0.0^{a} \\
(n=0 / 6) \text { N.D. }\end{array}$ & $\begin{array}{c}(\mathrm{n}=1 / 6) 2.84 \pm 0.0^{\mathrm{a}} \\
\quad(\mathrm{n}=0 / 6) \mathrm{N} . \mathrm{D} . \\
(\mathrm{n}=1 / 6) 2.00 \pm 0.0^{\mathrm{a}}\end{array}$ & $\begin{array}{c}(n=1 / 6) 2.00 \pm 0.0^{a} \\
(n=0 / 6) \text { N.D. } \\
(n=0 / 6) \text { N.D. }\end{array}$ & $\begin{array}{l}(\mathrm{n}=0 / 6) \text { N.D. } \\
(\mathrm{n}=0 / 6) \text { N.D. } \\
(\mathrm{n}=0 / 6) \text { N.D. }\end{array}$ \\
\hline Listeria monocytogenes & $\begin{array}{l}\mathrm{H}_{1} \\
\mathrm{H}_{2} \\
\mathrm{H}_{3} \\
\end{array}$ & $\begin{array}{l}(n=0 / 6) \text { N.D. } \\
(n=0 / 6) \text { N.D. } \\
(n=0 / 6) \text { N.D. }\end{array}$ & $\begin{array}{l}(n=0 / 6) \text { N.D. } \\
(n=0 / 6) \text { N.D. } \\
(n=0 / 6) \text { N.D. }\end{array}$ & $\begin{array}{l}(n=0 / 6) \text { N.D. } \\
(n=0 / 6) \text { N.D. } \\
(n=0 / 6) \text { N.D. }\end{array}$ & $\begin{array}{l}(n=0 / 6) \text { N.D. } \\
(n=0 / 6) \text { N.D. } \\
(n=0 / 6) \text { N.D. }\end{array}$ \\
\hline
\end{tabular}

$\mathrm{T}_{0}=$ day of packaging; $\mathrm{T}_{15}, \mathrm{~T}_{30}$ and $\mathrm{T}_{45}=$ respectively, 15,30 and 45 days of storage; $\mathrm{H}_{1}, \mathrm{H}_{2}$ and $\mathrm{H}_{3}$ indicate the smoking time respectively of 1,2 and 3 hours; means in the same row with different superscript letter indicate a significant difference $(\mathrm{P}<0.05)$ among days of storage; for each microbial group means in the same column with different superscript number indicate a significant difference $(\mathrm{P}<0.05)$ among smoking time. Values within brackets indicate the prevalence of positive samples. 
$0,15,30$ and 45 days) on physico-chemical and headspace gas composition, a General Linear Model was conducted using the Multifactor ANOVA procedure of Statgraphics Centurion XVI software (Stat Point Technologies).

\section{Results}

\section{Microbiological profile}

Total bacterial count ranged between $3.88 \pm 0.48 \log _{10} \mathrm{cfu} / \mathrm{g}$ at $\mathrm{T}_{0}$ and $3.24 \pm 1.02$ $\log _{10} \mathrm{cfu} / \mathrm{g}$ at $\mathrm{T}_{45}$. The bacterial count was significantly affected by smoking time $\left(\mathrm{P}<0.001 ; \mathrm{H}_{2}\right.$ showing values $0.49 \log _{10}$ lower than $\mathrm{H}_{1}$ ) and days of storage $\left(\mathrm{P}<0.001\right.$; a decrease of $1.22 \log _{10}$ was observed between $T_{0}$ and $T_{15}$ ) while the temperature of storage showed no effect $(\mathrm{P}>0.05)$. Yeast and molds were occasionally reported in the first fifteen days of storage, respectively in $2.6 \%\left(1.45 \pm 0.21 \log _{10}\right.$ $\mathrm{cfu} / \mathrm{g})$ and in $1.3 \%\left(2.00 \pm 0.0 \log _{10} \mathrm{cfu} / \mathrm{g}\right)$ of the samples. During the storage, the prevalence increased to $29.1 \%$ for yeast $\left(2.88 \pm 0.59 \log _{10} \mathrm{cfu} / \mathrm{g}\right)$ and to $22.2 \%$ for molds $\left(2.36 \pm 0.37 \log _{10} \mathrm{cfu} / \mathrm{g}\right)$. B. cereus was observed in four samples $(5.6 \%)$ stored at $4^{\circ} \mathrm{C}$ and in three samples $(4.1 \%)$ stored at $7^{\circ} \mathrm{C}$; mean count was $2.36 \pm 0.35 \log _{10} \mathrm{cfu} / \mathrm{g}$. Enterobacteriaceae were enumerated in one samples stored at $4^{\circ} \mathrm{C}$ for forty-five days (2.77 $\log _{10} \mathrm{cfu} / \mathrm{g}$ ) and in three samples stored at $7^{\circ} \mathrm{C}$ of which one for thirty days $\left(2.00 \log _{10} \mathrm{cfu} / \mathrm{g}\right.$ ) and two for forty-five days $\left(2.87 \pm 0.04 \quad \log _{10} \quad \mathrm{cfu} / \mathrm{g}\right)$. E. coli, Pseudomonas spp and Listeria monocytogenes were never detected. Tables 2 and 3 report the complete microbiological profile with mean counts $(\log 10 \mathrm{cfu} / \mathrm{g} ; \pm \mathrm{SD})$ over time by smoking time, respectively for $N S$ Ricotta Cheese stored at $4^{\circ} \mathrm{C}$ and $7^{\circ} \mathrm{C}$.

\section{Physicochemical and headspace gas composition}

The $\mathrm{pH}$ of ricotta salata cheese during refrigerated storage under vacuum packing decreased from an initial level $\left(\mathrm{T}_{0}\right)$ of $6.31 \pm 0.04$ to a final level $\left(\mathrm{T}_{45}\right)$ of $5.96 \pm 0.18$ $(\mathrm{P}<0.05)$; smoking time and days of storage showed a significant effect $(\mathrm{P}<0.05)$ while temperature of storage showed no impact on the $\mathrm{pH}(\mathrm{P}>0.05)$. The $\mathrm{a}_{\mathrm{W}}$ values ranged between $0.984 \pm 0.005$ at $\mathrm{T}_{0}$ and $0.983 \pm 0.005$ at $\mathrm{T}_{45}$; no significant difference was observed for smoking time and temperature of storage $(\mathrm{P}>0.05)$ while a significant effect of days of storage was observed, with higher values at $\mathrm{T}_{0}$ and $\mathrm{T}_{45}$ $(\mathrm{P}<0.05)$. Moisture ranged between $74.55 \pm 1.83 \%$ at $\mathrm{T}_{0}$ and $74.65 \pm 1.81 \%$ at $\mathrm{T}_{45}$, fat ranged between $11.13 \pm 1.43$ at $\mathrm{T}_{0}$ and $9.88 \pm 1.16$ at $\mathrm{T}_{45}$, proteins ranged between $10.36 \pm 0.53$ at $\mathrm{T}_{0}$ and $11.14 \pm 0.32$ at $\mathrm{T}_{45}$, salt content ranged between $1.87 \pm 0.34$ at $\mathrm{T}_{0}$ and $1.59 \pm 0.45$ at $\mathrm{T}_{45}$, respectively. Moisture was significantly affected by smoking time $(\mathrm{P}<0.001)$ with lower values observed in ricotta samples smoked for three hours, fat and proteins content were significantly affected $(\mathrm{P}<0.001)$ by days of storage while the salt content was significantly affected by smoking time $(\mathrm{P}<0.05)$ and days of storage $(\mathrm{P}<0.001)$. Smoking time and days of storage had a significant impact $(\mathrm{P}<0.001)$ on $\mathrm{N}, \mathrm{CO}_{2}$ and $\mathrm{O}_{2} \%$ headspace content while temperature of storage had a significant effect $(\mathrm{P}<0.05)$ on the $\mathrm{O}_{2} \%$ content (Tables 4 and 5).

\section{Discussion}

In recent years, the global interest for local foods has increased due to their perceived greater quality as compared to conventional foods (Aprile et al., 2016). In particular, traditional food products constitute an important element of European culture, identity and heritage (Almli et al., 2011; Vanhonacker et al., 2010). Consumer's attitude toward traditional foods is positive

Table 3. Evolution of the microbiological profile $\left(\log _{10} \mathrm{cfu} / \mathrm{g} ; \bar{x} \pm \mathrm{SD}\right)$ of MAP NS-Ricotta Cheese by smoking time during storage at $7^{\circ} \mathrm{C}$.

\begin{tabular}{|c|c|c|c|c|c|}
\hline \multirow[t]{2}{*}{ Microbial group } & \multirow[t]{2}{*}{ Smoking } & \multicolumn{3}{|c|}{ Day of storage } & \multirow[b]{2}{*}{$\mathrm{T}_{45}$} \\
\hline & & $\mathrm{T}_{0}$ & $\mathrm{~T}_{15}$ & $\mathrm{~T}_{30}$ & \\
\hline Aerobic mesophilic bacteria & $\begin{array}{l}\mathrm{H}_{1} \\
\mathrm{H}_{2} \\
\mathrm{H}_{3}\end{array}$ & $\begin{array}{l}(\mathrm{n}=6 / 6) 4.04 \pm 0.23^{\mathrm{al}} \\
(\mathrm{n}=6 / 6) 3.56 \pm 0.75^{\mathrm{al}} \\
(\mathrm{n}=6 / 6) 4.03 \pm 0.15^{\mathrm{al}}\end{array}$ & $\begin{array}{l}(\mathrm{n}=6 / 6) 2.79 \pm 0.31^{\mathrm{bl}} \\
(\mathrm{n}=6 / 6) 2.70 \pm 0.61^{\mathrm{al}} \\
(\mathrm{n}=6 / 6) 2.57 \pm 0.54^{\mathrm{bl}}\end{array}$ & $\begin{array}{l}(\mathrm{n}=6 / 6) 3.62 \pm 1.05^{\mathrm{abl}} \\
(\mathrm{n}=6 / 6) 2.75 \pm 0.83^{\mathrm{al}} \\
(\mathrm{n}=6 / 6) 2.94 \pm 0.82^{\mathrm{bl}}\end{array}$ & $\begin{array}{l}(\mathrm{n}=6 / 6) 3.48 \pm 0.82^{\mathrm{abl}} \\
(\mathrm{n}=6 / 6) 3.04 \pm 1.23^{\mathrm{al}} \\
(\mathrm{n}=6 / 6) 3.63 \pm 1.46^{\mathrm{abl}}\end{array}$ \\
\hline Enterobacteriaceae & $\begin{array}{l}\mathrm{H}_{1} \\
\mathrm{H}_{2} \\
\mathrm{H}_{3}\end{array}$ & $\begin{array}{l}(\mathrm{n}=0 / 6) \text { N.D. } \\
(\mathrm{n}=0 / 6) \text { N.D. } \\
(\mathrm{n}=0 / 6) \text { N.D. }\end{array}$ & $\begin{array}{l}(\mathrm{n}=0 / 6) \text { N.D. } \\
(\mathrm{n}=0 / 6) \text { N.D. } \\
(\mathrm{n}=0 / 6) \text { N.D. }\end{array}$ & $\begin{array}{c}(\mathrm{n}=1 / 6) 2.00 \pm 0.0 \\
(\mathrm{n}=0 / 6) \mathrm{N} . \mathrm{D} . \\
(\mathrm{n}=0 / 6) \mathrm{N} . \mathrm{D} .\end{array}$ & $\begin{array}{c}(\mathrm{n}=2 / 6) 2.87 \pm 0.04 \\
(\mathrm{n}=0 / 6) \text { N.D. } \\
(\mathrm{n}=0 / 6) \text { N.D. }\end{array}$ \\
\hline E. coli & $\begin{array}{l}\mathrm{H}_{1} \\
\mathrm{H}_{2} \\
\mathrm{H}_{3}\end{array}$ & $\begin{array}{l}(n=0 / 6) \text { N.D. } \\
(n=0 / 6) \text { N.D. } \\
(n=0 / 6) \text { N.D. }\end{array}$ & $\begin{array}{l}(n=0 / 6) \text { N.D. } \\
(n=0 / 6) \text { N.D. } \\
(n=0 / 6) \text { N.D. }\end{array}$ & $\begin{array}{l}(n=0 / 6) \text { N.D. } \\
(n=0 / 6) \text { N.D. } \\
(n=0 / 6) \text { N.D. }\end{array}$ & $\begin{array}{l}(n=0 / 6) \text { N.D. } \\
(n=0 / 6) \text { N.D. } \\
(n=0 / 6) \text { N.D. }\end{array}$ \\
\hline Yeast & $\begin{array}{l}\mathrm{H}_{1} \\
\mathrm{H}_{2} \\
\mathrm{H}_{3}\end{array}$ & $\begin{array}{l}(\mathrm{n}=0 / 6) \text { N.D. } \\
(\mathrm{n}=0 / 6) \text { N.D. } \\
(\mathrm{n}=0 / 6) \text { N.D. }\end{array}$ & $\begin{array}{l}(n=0 / 6) \text { N.D. } \\
(n=0 / 6) \text { N.D. } \\
(n=0 / 6) \text { N.D. }\end{array}$ & $\begin{array}{l}(\mathrm{n}=3 / 6) 2.73 \pm 0.60^{\mathrm{a}} \\
(\mathrm{n}=1 / 6) 3.17 \pm 0.00^{\mathrm{a}} \\
(\mathrm{n}=2 / 6) 2.60 \pm 0.43^{\mathrm{a}}\end{array}$ & $\begin{array}{c}(\mathrm{n}=3 / 6) 2.89 \pm 0.11^{\mathrm{a}} \\
(\mathrm{n}=0 / 6) \text { N.D. } \\
(\mathrm{n}=4 / 6) 3.16 \pm 1.09^{\mathrm{a}}\end{array}$ \\
\hline Molds & $\begin{array}{l}\mathrm{H}_{1} \\
\mathrm{H}_{2} \\
\mathrm{H}_{3}\end{array}$ & $\begin{array}{l}(n=0 / 6) \text { N.D. } \\
(n=0 / 6) \text { N.D. } \\
(n=0 / 6) \text { N.D. }\end{array}$ & $\begin{array}{c}(\mathrm{n}=1 / 6) 2.00 \pm 0.0 \mathrm{a} \\
(\mathrm{n}=0 / 6) \mathrm{N} . \mathrm{D} . \\
(\mathrm{n}=0 / 6) \text { N.D. }\end{array}$ & $\begin{array}{l}(\mathrm{n}=3 / 6) 2.10 \pm 0.17^{\mathrm{a}} \\
(\mathrm{n}=1 / 6) 2.00 \pm 0.00^{\mathrm{a}} \\
(\mathrm{n}=2 / 6) 2.45 \pm 0.64^{\mathrm{a}}\end{array}$ & $\begin{array}{c}(\mathrm{n}=2 / 6) 2.30 \pm 0.43^{\mathrm{a}} \\
(\mathrm{n}=0 / 6) \mathrm{N} \cdot \mathrm{D} . \\
(\mathrm{n}=0 / 6) \text { N.D. }\end{array}$ \\
\hline Pseudomonas spp & $\begin{array}{l}\mathrm{H}_{1} \\
\mathrm{H}_{2} \\
\mathrm{H}_{3}\end{array}$ & $\begin{array}{l}(n=0 / 6) \text { N.D. } \\
(n=0 / 6) \text { N.D. } \\
(n=0 / 6) \text { N.D. }\end{array}$ & $\begin{array}{l}(n=0 / 6) \text { N.D. } \\
(n=0 / 6) \text { N.D. } \\
(n=0 / 6) \text { N.D. }\end{array}$ & $\begin{array}{l}(n=0 / 6) \text { N.D. } \\
(n=0 / 6) \text { N.D. } \\
(n=0 / 6) \text { N.D. }\end{array}$ & $\begin{array}{c}(n=1 / 6) 3.36 \pm 0.0 \\
(n=0 / 6) \text { N.D. } \\
(n=0 / 6) \text { N.D. }\end{array}$ \\
\hline Bacillus cereus & $\begin{array}{l}\mathrm{H}_{1} \\
\mathrm{H}_{2} \\
\mathrm{H}_{3}\end{array}$ & $\begin{array}{c}(n=0 / 6) \text { N.D. } \\
(n=1 / 6) 2.60 \pm 0.00^{a} \\
(n=0 / 6) \text { N.D. }\end{array}$ & $\begin{array}{c}(n=0 / 6) \text { N.D. } \\
(n=0 / 6) \text { N.D. } \\
(n=1 / 6) 2.00 \pm 0.00^{a}\end{array}$ & $\begin{array}{c}(\mathrm{n}=0 / 6) \mathrm{N} . \mathrm{D} . \\
(\mathrm{n}=0 / 6) \mathrm{N} . \mathrm{D} . \\
(\mathrm{n}=1 / 6) 2.47 \pm 0.00^{\mathrm{a}}\end{array}$ & $\begin{array}{l}(\mathrm{n}=0 / 6) \text { N.D. } \\
(\mathrm{n}=0 / 6) \text { N.D. } \\
(\mathrm{n}=0 / 6) \text { N.D. }\end{array}$ \\
\hline Listeria monocytogenes & $\begin{array}{l}\mathrm{H}_{1} \\
\mathrm{H}_{2} \\
\mathrm{H}_{3}\end{array}$ & $\begin{array}{l}(n=0 / 6) \text { N.D. } \\
(n=0 / 6) \text { N.D. } \\
(n=0 / 6) \text { N.D. }\end{array}$ & $\begin{array}{l}(\mathrm{n}=0 / 6) \text { N.D. } \\
(\mathrm{n}=0 / 6) \text { N.D. } \\
(\mathrm{n}=0 / 6) \text { N.D. }\end{array}$ & $\begin{array}{l}(\mathrm{n}=0 / 6) \text { N.D. } \\
(\mathrm{n}=0 / 6) \text { N.D. } \\
(\mathrm{n}=0 / 6) \text { N.D. }\end{array}$ & $\begin{array}{l}(n=0 / 6) \text { N.D. } \\
(n=0 / 6) \text { N.D. } \\
(n=0 / 6) \text { N.D. }\end{array}$ \\
\hline
\end{tabular}

$\mathrm{T}_{0}=$ day of packaging; $\mathrm{T}_{15}, \mathrm{~T}_{30}$ and $\mathrm{T}_{45}=$ respectively, 15,30 and 45 days of storage; $\mathrm{H}_{1}, \mathrm{H}_{2}$ and $\mathrm{H}_{3}$ indicate the smoking time respectively of 1,2 and 3 hours; means in the same row with different superscript letter indicate a significant difference $(\mathrm{P}<0.05)$ among days of storage; for each microbial group means in the same column with different superscript number indicate a significant difference $(\mathrm{P}<0.05)$ among smoking time. Values within brackets indicate the prevalence of positive samples. 
since these goods are generally linked to regional identity and sensory quality (Guerrero et al., 2009). Introducing innovations into traditional food products seems somehow controversial since it has to improved production process on one hand and preserve the sensory quality seek by consumers on the other (Jordana, 2000). However, in order to maintain and expand the market of traditional food products food industries are now pursuing the further improvement in safety, health and convenience of such products by means of different innovations (Kühne et al., 2010). Food product packaging plays a key role in the innovation of traditional food products, contributing to capture potential consumer's attention and encouraging product purchase (Bloch, 1995; Silayoi and Speece, 2007; Piqueras-Fiszman and Spence, 2012). With regard to packaging, the main innovations introduced in the present study were the use of MAP packaging and the reduction of product size. The absence of the pressing step and the cold smoking instead of hot smoking were the main technological innovations in NS-Ricotta Cheese with respect to other traditional salted ricotta cheese. As consequence of the changes in the production process the novel smoked ricotta cheese has intrinsic properties $(\mathrm{pH}$ 6.2-6.3; $\mathrm{a}_{\mathrm{W}}$ 0.984-0.986) that are between the ricotta fresca $\left(\mathrm{pH} 6.5-6.8 ; \mathrm{a}_{\mathrm{W}} 0.990\right.$ 0.994 ) and ricotta salata ( $\mathrm{pH}$ 6.3-6.5; $\mathrm{a}_{\mathrm{W}}$ 0.950-0.980). The evolution during storage is similar to what has been observed in ricotta salata with a $0.4-0.5 \mathrm{pH}$ decrease (Casti et al., 2016) while $\mathrm{a}_{\mathrm{W}}$, similarly to both ricotta fresca and ricotta salata remained stable or with little variation (Pala et al., 2016; Spanu et al., 2013). Food safety criteria (L. monocytogenes) and process hygiene criteria (E. coli) were always compliant with Regulation (EC) No 2073/2005 (European Commission, 2005). Enterobacteriaceae were enumerated only in one sample (1.4\%) after 45 days of storage at $4^{\circ} \mathrm{C}$ and in three samples $(4.2 \%)$ after 30 days at $7^{\circ} \mathrm{C}$, with mean levels always below $3 \log _{10} \mathrm{cfu} / \mathrm{g}$. These values are lower as compared to ricotta fresca and ricotta salata where mean counts of ca. 6-7 $\log _{10}$ $\mathrm{cfu} / \mathrm{g}$ and of ca. 4-5 $\log 10 \mathrm{cfu} / \mathrm{g}$ were observed respectively after 21 and 60 days of refrigerated storage (Casti et al., 2016; Pala et al., 2016). Despite no microbiologi- cal criteria has been defined for Enterobacteriaceae in whey cheeses that undergone heat treatment, these are important indicator microorganisms, revealing good hygienic conditions during the manufacturing process. B. cereus was reported occasionally, with counts of ca. $2 \log _{10}$ $\mathrm{cfu} / \mathrm{g}$, dose below the five to eight $\log _{10}$ $\mathrm{cfu} / \mathrm{g}$, generally considered necessary to cause illness (ICMSF, 1996). B. cereus vegetative cells were not recovered from samples stored up to 45 days at both $4^{\circ} \mathrm{C}$ and $7^{\circ} \mathrm{C}$. This finding is in agreement with previous investigation conducted on salted ricotta samples, where vegetative cells decreased during refrigerated storage, indicating that the contamination was likely due to mesophilic strains which minimum growth temperature is $15^{\circ} \mathrm{C}$ (Spanu et al., 2016). Pseudomonas spp. were never detectable, despite fresh ricotta cheese is particularly susceptible of secondary contamination and represents an excellent substrate for the growth of psychotropic spoilage microorganisms (Ibba et al., 2013; Scarano et al., 2014; Spanu et al., 2015c). The present finding is in contrast with previous investigation where Pseudomonas

Table 4. $\mathrm{pH}, \mathrm{a}_{\mathrm{W}}$, physico-chemical and headspace gas composition $(\bar{x} \pm \mathrm{SD})$ of ricotta samples by smoking time $(1,2$ and 3 hours $)$ stored at $4^{\circ} \mathrm{C}$.

\begin{tabular}{|c|c|c|c|c|c|}
\hline \multirow[t]{2}{*}{ Parameters } & \multirow[t]{2}{*}{ Smoking } & \multicolumn{3}{|c|}{ Day of storage } & \multirow[b]{2}{*}{$\mathrm{T}_{45}$} \\
\hline & & $\mathrm{T}_{0}$ & $\mathrm{~T}_{15}$ & $\mathrm{~T}_{30}$ & \\
\hline $\mathrm{pH}$ & $\begin{array}{l}\mathrm{H}_{1} \\
\mathrm{H}_{2} \\
\mathrm{H}_{3}\end{array}$ & $\begin{array}{l}(\mathrm{n}=6) 6.32 \pm 0.03^{\mathrm{al}} \\
(\mathrm{n}=6) 6.29 \pm 0.03^{\mathrm{al}} \\
(\mathrm{n}=6) 6.32 \pm 0.06^{\mathrm{al}}\end{array}$ & $\begin{array}{l}(\mathrm{n}=6) 6.26 \pm 0.15^{\mathrm{abl}} \\
(\mathrm{n}=6) 6.13 \pm 0.19^{\mathrm{bl}} \\
(\mathrm{n}=6) 6.05 \pm 0.27^{\mathrm{bl}}\end{array}$ & $\begin{array}{l}(\mathrm{n}=6) 6.15 \pm 0.07^{\mathrm{bl}} \\
(\mathrm{n}=6) 5.94 \pm 0.87^{\mathrm{c} 2} \\
(\mathrm{n}=6) 5.81 \pm 1.33^{\mathrm{c} 3}\end{array}$ & $\begin{array}{l}(\mathrm{n}=6) 6.18 \pm 0.68^{\mathrm{bl}} \\
(\mathrm{n}=6) 5.96 \pm 0.11^{\mathrm{c} 2} \\
(\mathrm{n}=6) 5.80 \pm 0.13^{\mathrm{c} 3}\end{array}$ \\
\hline$a_{w}$ & $\begin{array}{l}\mathrm{H}_{1} \\
\mathrm{H}_{2} \\
\mathrm{H}_{3}\end{array}$ & $\begin{array}{l}(\mathrm{n}=6) 0.984 \pm 0.004^{\mathrm{al}} \\
(\mathrm{n}=6) 0.984 \pm 0.005^{\mathrm{al}} \\
(\mathrm{n}=6) 0.986 \pm 0.005^{\mathrm{al}}\end{array}$ & $\begin{array}{l}(\mathrm{n}=6) 0.981 \pm 0.005^{\mathrm{al}} \\
(\mathrm{n}=6) 0.980 \pm 0.006^{\mathrm{al}} \\
(\mathrm{n}=6) 0.979 \pm 0.006^{\mathrm{bl}}\end{array}$ & $\begin{array}{l}(\mathrm{n}=6) 0.980 \pm 0.004^{\mathrm{al}} \\
(\mathrm{n}=6) 0.979 \pm 0.005^{\mathrm{al}} \\
(\mathrm{n}=6) 0.980 \pm 0.003^{\mathrm{bl}}\end{array}$ & $\begin{array}{l}(\mathrm{n}=6) 0.982 \pm 0.004^{\mathrm{al}} \\
(\mathrm{n}=6) 0.982 \pm 0.005^{\mathrm{al}} \\
(\mathrm{n}=6) 0.983 \pm 0.004^{\mathrm{ab}}\end{array}$ \\
\hline Moisture (\%) & $\begin{array}{l}\mathrm{H}_{1} \\
\mathrm{H}_{2} \\
\mathrm{H}_{3}\end{array}$ & $\begin{array}{l}(\mathrm{n}=6) 75.08 \pm 1.17^{\mathrm{al}} \\
(\mathrm{n}=6) 74.92 \pm 2.29^{\mathrm{al}} \\
(\mathrm{n}=6) 73.66 \pm 1.92^{\mathrm{al}}\end{array}$ & $\begin{array}{l}(\mathrm{n}=6) 75.12 \pm 2.61^{\mathrm{al}} \\
(\mathrm{n}=6) 74.92 \pm 2.03^{\mathrm{al}} \\
(\mathrm{n}=6) 73.92 \pm 1.46^{\mathrm{al}}\end{array}$ & $\begin{array}{l}(\mathrm{n}=6) 74.91 \pm 0.60^{\mathrm{al}} \\
(\mathrm{n}=6) 74.78 \pm 1.73^{\mathrm{al}} \\
(\mathrm{n}=6) 74.47 \pm 1.22^{\mathrm{al}}\end{array}$ & $\begin{array}{l}(\mathrm{n}=6) 75.00 \pm 1.63^{\mathrm{a}} \\
(\mathrm{n}=6) 75.48 \pm 1.91^{\mathrm{al}} \\
(\mathrm{n}=6) 73.95 \pm 2.05^{\mathrm{a} 1}\end{array}$ \\
\hline Fat (\%) & $\begin{array}{l}\mathrm{H}_{1} \\
\mathrm{H}_{2} \\
\mathrm{H}_{3}\end{array}$ & $\begin{array}{l}(\mathrm{n}=6) 10.89 \pm 1.01^{\mathrm{al}} \\
(\mathrm{n}=6) 10.71 \pm 1.52^{\mathrm{al}} \\
(\mathrm{n}=6) 11.80 \pm 1.73^{\mathrm{al}}\end{array}$ & $\begin{array}{l}(\mathrm{n}=6) 10.49 \pm 2.00^{\mathrm{al}} \\
(\mathrm{n}=6) 10.11 \pm 1.87^{\mathrm{al}} \\
(\mathrm{n}=6) 10.41 \pm 1.05^{\mathrm{abl}}\end{array}$ & $\begin{array}{l}(\mathrm{n}=6) 10.64 \pm 1.09^{\mathrm{al}} \\
(\mathrm{n}=6) 9.83 \pm 0.98^{\mathrm{al}} \\
(\mathrm{n}=6) 9.55 \pm 0.74^{\mathrm{bl}}\end{array}$ & $\begin{array}{c}(\mathrm{n}=6) 10.46 \pm 0.95^{\mathrm{al}} \\
(\mathrm{n}=6) 9.16 \pm 1.72^{\mathrm{al}} \\
(\mathrm{n}=6) 10.26 \pm 1.15^{\mathrm{bl}}\end{array}$ \\
\hline Proteins (\%) & $\begin{array}{l}\mathrm{H}_{1} \\
\mathrm{H}_{2} \\
\mathrm{H}_{3}\end{array}$ & $\begin{array}{l}(\mathrm{n}=6) 10.34 \pm 0.53^{\mathrm{al}} \\
(\mathrm{n}=6) 10.34 \pm 0.58^{\mathrm{al}} \\
(\mathrm{n}=6) 10.40 \pm 0.61^{\mathrm{al}}\end{array}$ & $\begin{array}{l}(\mathrm{n}=6) 10.89 \pm 0.38^{\mathrm{bl}} \\
(\mathrm{n}=6) 10.86 \pm 0.24^{\mathrm{bl}} \\
(\mathrm{n}=6) 10.88 \pm 0.56^{\mathrm{bl}}\end{array}$ & $\begin{array}{l}(\mathrm{n}=6) 10.90 \pm 0.48^{\mathrm{bl}} \\
(\mathrm{n}=6) 10.98 \pm 0.34^{\mathrm{bl}} \\
(\mathrm{n}=6) 11.30 \pm 0.19^{\mathrm{bl}}\end{array}$ & $\begin{array}{l}(\mathrm{n}=6) 11.01 \pm 0.32^{\mathrm{bl}} \\
(\mathrm{n}=6) 11.20 \pm 0.47^{\mathrm{bl}} \\
(\mathrm{n}=6) 11.03 \pm 0.56^{\mathrm{bl}}\end{array}$ \\
\hline Salt (\%) & $\begin{array}{l}\mathrm{H}_{1} \\
\mathrm{H}_{2} \\
\mathrm{H}_{3}\end{array}$ & $\begin{array}{l}(\mathrm{n}=6) 1.91 \pm 0.43^{\mathrm{al}} \\
(\mathrm{n}=6) 1.99 \pm 0.36^{\mathrm{al}} \\
(\mathrm{n}=6) 1.73 \pm 0.21^{\mathrm{al}}\end{array}$ & $\begin{array}{l}(\mathrm{n}=6) 1.84 \pm 0.38^{\mathrm{al}} \\
(\mathrm{n}=6) 1.82 \pm 0.66^{\mathrm{al}} \\
(\mathrm{n}=6) 1.77 \pm 0.38^{\mathrm{al}}\end{array}$ & $\begin{array}{l}(\mathrm{n}=6) 1.77 \pm 0.24^{\mathrm{al}} \\
(\mathrm{n}=6) 1.70 \pm 0.49^{\mathrm{al}} \\
(\mathrm{n}=6) 1.56 \pm 0.22^{\mathrm{al}}\end{array}$ & $\begin{array}{l}(\mathrm{n}=6) 1.74 \pm 0.57^{\mathrm{al}} \\
(\mathrm{n}=6) 1.65 \pm 0.47^{\mathrm{al}} \\
(\mathrm{n}=6) 1.48 \pm 0.22^{\mathrm{al}}\end{array}$ \\
\hline $\mathrm{N}(\%)$ & $\begin{array}{l}\mathrm{H}_{1} \\
\mathrm{H}_{2} \\
\mathrm{H}_{3}\end{array}$ & $\begin{array}{l}(\mathrm{n}=4) 90.22 \pm 1.85^{\mathrm{al}} \\
(\mathrm{n}=4) 89.48 \pm 2.02^{\mathrm{al}} \\
(\mathrm{n}=3) 87.43 \pm 2.76^{\mathrm{al}}\end{array}$ & $\begin{array}{l}(\mathrm{n}=6) 93.98 \pm 0.84^{\mathrm{b} 1} \\
(\mathrm{n}=4) 92.28 \pm 0.91^{\mathrm{b} 2} \\
(\mathrm{n}=6) 92.75 \pm 0.79^{\mathrm{b} 2}\end{array}$ & $\begin{array}{l}(\mathrm{n}=5) 93.98 \pm 1.13^{\mathrm{bl}} \\
(\mathrm{n}=6) 93.67 \pm 0.48^{\mathrm{bl}} \\
(\mathrm{n}=5) 93.22 \pm 0.52^{\mathrm{bl}}\end{array}$ & $\begin{array}{l}(\mathrm{n}=6) 94.33 \pm 0.71^{\mathrm{bl}} \\
(\mathrm{n}=6) 93.67 \pm 0.84^{\mathrm{bl2}} \\
(\mathrm{n}=6) 92.95 \pm 0.76^{\mathrm{b} 2}\end{array}$ \\
\hline $\mathrm{O}_{2}(\%)$ & $\begin{array}{l}\mathrm{H}_{1} \\
\mathrm{H}_{2} \\
\mathrm{H}_{3}\end{array}$ & $\begin{array}{l}(\mathrm{n}=4) 1.36 \pm 0.18^{\mathrm{al}} \\
(\mathrm{n}=4) 1.12 \pm 0.27^{\mathrm{al}} \\
(\mathrm{n}=3) 1.08 \pm 0.43^{\mathrm{al}}\end{array}$ & $\begin{array}{l}(\mathrm{n}=6) 0.90 \pm 0.35^{\mathrm{al}} \\
(\mathrm{n}=4) 0.59 \pm 0.11^{\mathrm{b} 12} \\
(\mathrm{n}=6) 0.42 \pm 0.20^{\mathrm{b} 2}\end{array}$ & $\begin{array}{l}(\mathrm{n}=5) 0.95 \pm 0.92^{\mathrm{al}} \\
(\mathrm{n}=6) 0.17 \pm 0.12^{\mathrm{c} 2} \\
(\mathrm{n}=5) 0.11 \pm 0.11^{\mathrm{c} 2}\end{array}$ & $\begin{array}{l}(\mathrm{n}=6) 0.24 \pm 0.37^{\mathrm{bl}} \\
(\mathrm{n}=6) 0.13 \pm 0.11^{\mathrm{cl} 2} \\
(\mathrm{n}=6) 0.06 \pm 0.03^{\mathrm{c} 2}\end{array}$ \\
\hline $\mathrm{CO}_{2}(\%)$ & $\begin{array}{l}\mathrm{H}_{1} \\
\mathrm{H}_{2} \\
\mathrm{H}_{3}\end{array}$ & $\begin{array}{l}(\mathrm{n}=4) 8.42 \pm 1.89^{\mathrm{al}} \\
(\mathrm{n}=4) 9.55 \pm 2.14^{\mathrm{al}} \\
(\mathrm{n}=3) 11.05 \pm 2.35^{\mathrm{al}}\end{array}$ & $\begin{array}{l}(\mathrm{n}=6) 5.15 \pm 0.59^{\mathrm{bl}} \\
(\mathrm{n}=4) 7.12 \pm 0.88^{\mathrm{b} 2} \\
(\mathrm{n}=6) 6.82 \pm 0.81^{\mathrm{b} 2}\end{array}$ & $\begin{array}{l}(\mathrm{n}=5) 5.02 \pm 0.56^{\mathrm{b} 1} \\
(\mathrm{n}=6) 6.18 \pm 0.40^{\mathrm{b} 2} \\
(\mathrm{n}=5) 6.72 \pm 0.44^{\mathrm{b} 2}\end{array}$ & $\begin{array}{l}(\mathrm{n}=6) 5.42 \pm 0.62^{\mathrm{bl}} \\
(\mathrm{n}=6) 6.15 \pm 0.82^{\mathrm{b} 12} \\
(\mathrm{n}=6) 7.00 \pm 0.72^{\mathrm{b} 2}\end{array}$ \\
\hline
\end{tabular}

Values in the same row with different superscript letter are statistically different $(\mathrm{P}<0.05)$. Values in the same column with different superscript number are statistically different $(\mathrm{P}<0.05)$. 
spp. grew from the initial contamination of ca. two $\log _{10} \mathrm{cfu} / \mathrm{g}$ to as high as $7 \log _{10}$ $\mathrm{cfu} / \mathrm{g}$ after 21 days of refrigerated storage (Pala et al., 2016; Spanu et al., 2017, 2018). The aerobic mesophilic bacteria from ca. four $\log _{10} \mathrm{cfu} / \mathrm{g}$ observed at the day of packaging decreased to $<3 \log _{10}$ $\mathrm{cfu} / \mathrm{g}$ after fifteen days of storage, then slowly increased (ca. $0.5 \log _{10} \mathrm{cfu} / \mathrm{g}$ ) at 45 days to levels always below the initial contamination. These results are different as compared to other sheep ricotta cheeses. In MAP fresh ricotta cheese aerobic mesophilic count increased from levels $<3$ $\log _{10} \mathrm{cfu} / \mathrm{g}$ up to $7-8 \log _{10} \mathrm{cfu} / \mathrm{g}$ after 21 days of refrigerated storage, largely accountable to the growth of psychotropic microorganisms such as Pseudomonas spp. (Pala et al., 2016 Spanu et al., 2017). This difference is explained by the absence in the novel smoked ricotta cheese of Pseudomonas spp. contamination, further indication that GHP were strictly observed during the manufacturing process. In traditional salted ricotta cheese (i.e. ricotta toscanella) the mean aerobic mesophilic bacteria count on the rind surface was ca. 7-8 $\log _{10} \mathrm{cfu} / \mathrm{g}$ before vacuum packaging
(Spanu et al., 2013; Casti et al., 2016). Higher counts of salted ricotta are justified by the phases of pressing, dry salting and maturation conducted with the exposed product which increase the risk of cross contamination originating from the processing environment (Ibba et al., 2013). A progressive reduction of $\mathrm{O}_{2} \%$ concentration in the headspace was observed during storage, indicating the growth of aerobic microorganism, mostly yeast and molds. Instead, the reduction in $\mathrm{CO}_{2} \%$ concentration is the results of gas solving in the product. MAP packaging is used in fresh ricotta cheese, showing an initial increase of $\mathrm{O}_{2} \%$ concentration, followed by a successive decrease (Pala et al., 2016; Spanu et al., 2017, 2018). This could be explained with the different size of fresh ricotta compared to NS-Ricotta Cheese (1.1-1.7 kg vs. $300 \mathrm{~g}$ ) and therefore the less favorable headspace/food ratio. The greater amount of $\mathrm{O}_{2}$ incorporated in the ricotta fresca food matrix, which is successively released into the headspace, greatly impact on the time needed to reach the equilibrium condition of the gas mixture (Simpson and Carevic, 2004).

\section{Conclusions}

In the frame of an increasing globalization of food market, innovation is an essential strategy for small and medium sized enterprises to achieve a competitive advantage versus global foods (Murphy, 2002; Avermaete et al., 2004; Gellynck et al., 2007; Albayrak and Gunes, 2010). However, conjugate innovation and tradition is a complex task due to the controversy involved (Jordana, 2000). The present study demonstrated the feasibility of introducing product innovation in a traditional dairy product of Sardinia such as ricotta cheese. Despite the microbiological results obtained in the present study indicate a general safety of the product, definitive conclusion should be supported by specific challenge test. In fact, the intrinsic properties describe a product supporting the possible growth of spoilage and pathogens microorganism. Under the defined refrigerated storage conditions, psychotropic microorganisms such as Pseudomonas spp, Listeria monocytogenes and eventual $B$. cereus psychotropic strains represent major

Table 5. $\mathrm{pH}, \mathrm{a}_{\mathrm{W}}$, physico-chemical and headspace gas composition $(\bar{x} \pm \mathrm{SD})$ of ricotta samples by smoking time $(1,2$ and 3 hours $)$ stored at $7^{\circ} \mathrm{C}$.

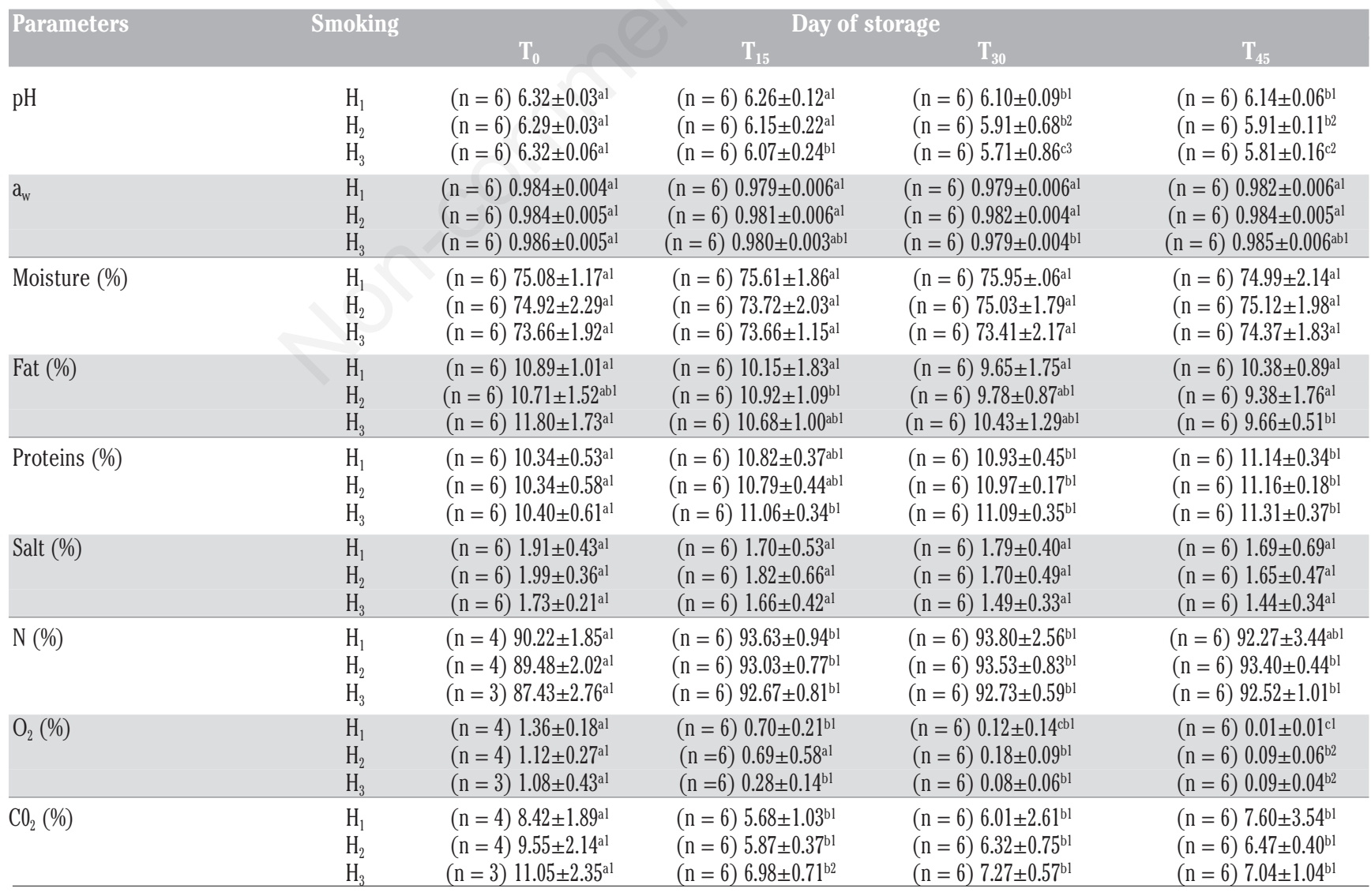

Values in the same row with different superscript letter are statistically different $(\mathrm{P}<0.05)$. Values in the same column with different superscript number are statistically different $(\mathrm{P}<0.05)$. 
concerns. The salted and smoked ricotta cheese is a food that should be regarded at high risk of secondary contamination; therefore, it is essential the strict implementation of good hygienic practices especially in the production steps between curd rising and packaging.

\section{References}

Albayrak M, Gunes E, 2010. Traditional foods: Interaction between local and global foods in Turkey. Afr J Business Manag 4:555.

Almli VL, Verbeke W, Vanhonacker F, Næs T, Hersleth M, 2011. General image and attribute perceptions of traditional food in six European countries. Food Qual Prefer 22:129-38.

Aprile MC, Caputo V, Nayga Jr RM, 2016. Consumers' preferences and attitudes toward local food products. J Food Prod Market 22:19-42.

Avermaete T, Viaene J, Morgan EJ, Crawford $\mathrm{N}, 2004$. The impact of firm characteristics and macroeconomic performance on innovation in small food firms: Case study from Belgium, Ireland and UK. IN: Innovation in Small Firms and Dynamics of Local Development. Eds: T. de Noronha Vaz, J. Viaene and M. Wigier. Warsaw: Scholar Publishing House, 7995.

Bloch PH, 1995. Seeking the ideal form: Product design and consumer response. J Market 16-29.

CAC (Codex Alimentarius Commission), 1995. Codex standard for whey powders. CODEX STAN 289-1995. Rev. 2003. Amendment 2006, 2010.

Casti D, Scarano C, Pala CC, Cossu F, Lamon S, Spanu V, Ibba M, Mocci AM, Tedde F, Nieddu G, Spanu C, De Santis EPL, 2016. Evolution of the microbiological profile of vacuum-packed ricotta salata cheese during shelf-life. Ital J Food Saf 5:5501

European Commission, 2005. Commission Regulation of 15 November 2005 on microbiological criteria for foodstuffs, 2073/2005/EC. In: Official Journal, L 338/1, 22/12/2005.

FAOSTAT, FAO, 2018. Food and Agriculture Organization of the united Nations Statistics Division. Rome: FAO. Available from: http://www.fao.org/dairy-production-products/products/types-and-characteristics/en/. Accessed June 6th 2018.

Gellynck X, Kühne B, 2008. Innovation and collaboration in traditional food chain networks. J Chain Network Sci 8:121-9.

Gellynck X, Vermeire B, Viaene J, 2007. Innovation in food firms: contribution of regional networks within the international business context. Entrepreneur Reg Dev 19:209-26.

Guerrero L, Guàrdia MD, Xicola J, Verbeke W, Vanhonacker F, Zakowska-Biemans S, Sajdakowska M, Sulmont-Rossé C, Issanchou S, Contel M, Scalvedi ML, Granli BS, Scalvedi ML, 2009. Consumer-driven definition of traditional food products and innovation in traditional foods. A qualitative cross-cultural study. Appetite 52:345-54.

Ibba M, Cossu F, Spanu V, Virdis S, Spanu C, Scarano C, De Santis EP, 2013. Listeria monocytogenes contamination in dairy plants: evaluation of Listeria monocytogenes environmental contamination in two cheese-making plants using sheeps milk. Ital J Food Saf 2:e31.

ICMSF, 1996. International commission on microbiological specifications for foods. Micro-organisms in foods. In: Microbiological Specification of Food Pathogens, vol. 5. Blackie Academic and Professional, London, 514 pp.

Jordana J, 2000. Traditional foods: challenges facing the European food industry. Food Res Int 33:147-52.

Kühne B, Vanhonacker F, Gellynck X, Verbeke W, 2010. Innovation in traditional food products in Europe: Do sector innovation activities match consumers' acceptance? Food Qual Pref 21:629-38.

Lipan L, Sánchez-Rodríguez L, CanoLamadrid M, Collado-González J, Noguera-Artiaga L, Sendra E, CarbonellBarrachina AA, 2017. Modernisation of traditional food processes and products. In Consumer trends and new product opportunities in the food sector (pp. 901-905). Wageningen Academic Publishers.

Man CD, Jones AA, Eds., 1994. Shelf life evaluation of foods. Glasgow: Blackie Academic \& Professional.

McElhatton A, El Idrissi MM, Eds., 2016. Modernization of traditional food processes and products (Vol. 11). Springer.

Murphy M, 2002. Organisational change and firm performance. OECD Science Technol Industry Working Papers (14), doi:10.1787/615168153531

Pala C, Scarano C, Venusti M, Sardo D, Casti D, Cossu F, Lamon S, Spanu V, Ibba M, Marras M, Paba A, Spanu C, De Santis EPL, 2016. Shelf life evaluation of ricotta fresca sheep cheese in modified atmosphere packaging. Ital J Food Saf 5:5502.

Piqueras-Fiszman B, Spence C, 2012. The weight of the container influences expected satiety, perceived density, and subsequent expected fullness. Appetite 58:55962.

Pulinas L, Spanu C, Idda I, Ibba I, Nieddu G, Virdis S, Scarano C, Piras F, Spano N, Sanna G, De Santis EPL, 2017.
Production of farmstead lactose-free Pecorino di Osilo and ricotta cheeses from sheep's milk. Ital J Food Saf 6:6353.

Silayoi P, Speece M, 2007. The importance of packaging attributes: a conjoint analysis approach. Eur J Market 41:1495-517.

Simpson R, Carevic E, 2004. Designing a modified atmosphere packaging system for foodservice portions on nonrespiring foods: optimal gas mixture and food/headspace ratio. Foodserv Res Int 14:257-72.

Scarano C, Giacometti F, Manfreda G, Lucchi A, Pes E, Spanu C, De Santis EP, Serraino A, 2014. Arcobacter butzleri in sheep ricotta cheese at retail and related sources of contamination in an industrial dairy plant. Appl Environ Microbiol 80:703641.

Spanu C, Spanu V, Pala C, Virdis S, Scarano C, De Santis EPL, 2013. Evaluation of a post-lethality treatment against Listeria monocytogenes on Ricotta salata cheese. Food Control 200:205-30.

Spanu C, Scarano C, Spanu V, Pala C, Di Salvo R, Piga C, Buschettu L, Casti D, Lamon S, Cossu F, Ibba M, De Santis EPL, 2015a. Comparison of post-lethality thermal treatment conditions on the reduction of Listeria monocytogenes and sensory properties of vacuum packed ricotta salata cheese. Food Control 50:740-7.

Spanu C, Scarano C, Spanu V, Pala C, Salvo RD, Piga C, Ullu A, Casti D, Lamon S, Cossu F, Ibba M, De Santis EPL, 2015 b. Inactivation of Listeria monocytogenes using water bath heat treatment in vacuum packed Ricotta Salata cheese wedges. J Food Sci 80:M1549-56.

Spanu C, Scarano C, Ibba M, Spanu V, De Santis EPL, 2015c. Occurrence and traceability of Listeria monocytogenes strains isolated from sheep's milk cheese-making plants environment. Food Control 47:31825.

Spanu C, Scarano C, Spanu V, Pala C, Casti D, Lamon S, Cossu F, Ibba M, Nieddu G, De Santis EP, 2016. Occurrence and behavior of Bacillus cereus in naturally contaminated ricotta salata cheese during refrigerated storage. Food Microbiol 58:135-8.

Spanu C, Scarano C, Piras F, Spanu V, Pala C, Casti D, Lamon S, Cossu F, Ibba M, Nieddu G, De Santis EPL, 2017. Testing commercial biopreservative against spoilage microorganisms in MAP packed Ricotta fresca cheese. Food Microbiol 66:72-6.

Spanu C, Piras F, Mocci AM, Nieddu G, De Santis EPL, Scarano C, 2018. Use of Carnobacterium spp protective culture in MAP packed Ricotta fresca cheese to control Pseudomonas spp. Food Microbiol 74:50-6.

Storelli MM, Scarano C, Spanu C, De Santis 
EPL, Busco VP, Storelli A, Marcotrigiano GO, 2012. Levels and congener profiles of polychlorinated dibenzo-p-dioxins (PCDDs), polychlorinated dibenzofurans (PCDFs) and polychlorinated biphenyls (PCBs) in sheep milk from an industri- alised area of Sardinia, Italy. Food Chem Toxicol 50:1413-7.

Troiani C, 2015. Il siero di latte è una risorsa per diversi mercati. 16 January 2015. Available from: http://www.lattenews.it/ilsiero-di-latte-e-una-risorsa-per-diversi- mercati/

Vanhonacker F, Lengard V, Hersleth M, Verbeke W, 2010. Profiling European traditional food consumers. Br Food $\mathrm{J}$ 112:871-86. 